\title{
Smoking and its effect on scar healing
}

\author{
A. E. K. Deliaert • E. Van den Kerckhove • S. Tuinder • \\ S. M. J. S. Noordzij • T. S. Dormaar • \\ R. R. W. J. van der Hulst
}

Received: 26 June 2011 / Accepted: 9 November 2011 / Published online: 3 January 2012

(C) The Author(s) 2011. This article is published with open access at Springerlink.com

\begin{abstract}
Scar formation is influenced by several factors such as wound infection, tension, wound depth and anatomical localization. Hypertrophic scarring is often the result of an imbalance in the wound and scar healing process. The exact underlying pathophysiological mechanism remains unclear. Smoking has a higher risk of postoperative complications probably due to a diminished macrophage induction. Following our clinical impression that smokers without postoperative wound infections show esthetically better scars, we evaluated the scars after a reduction mammaplasty in smoking and nonsmoking patients in a prospective clinical trial. Between July 2006 and September 2007, 13 smokers and 30 non smokers with a reduction mammaplasty were included. They were recruited from Viecuri Medical Centre and Atrium
\end{abstract}

A. E. K. Deliaert $(\bowtie) \cdot$ E. Van den Kerckhove $\cdot S$. Tuinder

R. R. W. J. van der Hulst

Department of Plastic Surgery,

Maastricht University Medical Centre,

P.O. Box 5800, 6202 AZ Maastricht, The Netherlands

e-mail: andeliaert8@hotmail.com

E. Van den Kerckhove

Department of Rehabilitation Sciences and Kinesiology,

Katholieke Universiteit Leuven,

Leuven, Belgium

E. Van den Kerckhove

Department of Physical Medicine and Rehabilitation and Burns

Center, Katholieke Universiteit Leuven,

Leuven, Belgium

S. M. J. S. Noordzij

Department of Radiology, Maastricht University Medical Centre,

Maastricht, The Netherlands

T. S. Dormaar • R. R. W. J. van der Hulst

Department of Plastic Surgery Viecuri Medical Centre,

Venlo, The Netherlands
Medical Centre in the Netherlands after written consent. Surgical data and data of the patients' condition were collected. Follow-up for erythema values of the scars was done with a colorimeter (The Minolta CR-300, Minolta Camera Co., Ltd., Osaka Japan) at 1, 3, 6 and 9 months postoperatively on four standardized postsurgical sites. ANOVA and Chi-square test were used for statistical analysis. In the smoking group, the scars were significantly less red compared to the nonsmoking group. No significant differences were found in BMI, resection weight and drain production between both groups. Although smoking is certainly not recommended as a preventive therapy to influence scar healing, this study confirms our assumption that smokers tend to have faster and less erythemateous scar healing to nonsmokers. Further research is needed to understand the mechanism of the effect of smoking on scars.

Keywords Hypertrophic scarring · Smoking · Breast reduction surgery $\cdot$ Scarring $\cdot$ Postoperative complications after breast reduction surgery. Erythema

\section{Introduction}

Wound healing and the subsequent scar formation require an accurate balance of inflammation [1]. Smoking is known to result in more wound related postoperative complications, probably by disturbing the normal inflammatory physiology. Cooperating cellular responses have to be appropriate in several phases of wound healing. Diminished function of the neutrophils and macrophages results in chronic nonhealing wounds, e.g., in diabetes [2]. Hypertrophic scarring, on the other hand, can be the result of an exaggerated inflammatory response. The production of certain subspecies of TGF- $\beta$ by the macrophages seems to 
be important in inducing hypertrophic scarring [3]. Local discomfort such as pruritus, pain, restricted mobility and aesthetic impairment result in a reduced quality of life [4]. Hypertrophic scars often arise after burn wounds and appear 1 to 3 months after trauma. Our clinical impression is that smokers, although having complicated or delayed wound healing, almost never present with hypertrophic scars. Therefore, we hypothesize that smoking has a positive influence on postoperative scarring due to its effect on the inflammatory response. To evaluate this clinical impression, we performed a prospective patient-controlled study comparing postoperative scar activity in smoking and nonsmoking women undergoing a reduction mammaplasty.

\section{Materials and methods}

A double center, prospective study was performed in female patients undergoing reduction mammaplasty. All patients between the ages of 17 and 70 years with at least a DD cup were included in the study. Inclusion was done between July 2006 and September 2007 at Viecuri Medical Centre in Venlo and Atrium Medical Centre in Heerlen, both situated in the Netherlands. The study was approved by the ethical committees of both hospitals and written informed consent was obtained from each patient. Patients were referred to the Department of Plastic Surgery by the general practioner because of complaints of breast hypertrophy such as intertrigo and head, neck and/or shoulder complaints. The indication to perform a reduction mammaplasty was made by the plastic surgeon. Exclusion criteria were the presence of diabetes, skin disorders, use of corticosteroids and immunosupressive medication, history of keloids or hypertrophic scarring and degenerative or metabolic disorders.

Surgery was performed by a plastic surgeon or a senior resident using a mediocranial pedicle technique. There was no prophylactic use of antibiotics. Data on the condition of the patient (body mass index, age) and surgical data (reduction weight) were collected. One day after surgery, the patients were dismissed from the hospital. One month postoperatively, the patient started to use a hydrating crème on the scar. Follow-up took place at 1, 3, 6 and 9 months after reduction mammaplasty. Dehiscence was defined as every spontaneous disruption of the wound occurring during the first 3 months postoperatively. Each wound dehiscence independent of size was registered. Coloric measurement was not performed in case of dehiscence at this particular area. In case of clinical signs of infection (secretion, fever, erythema), antibiotics were given. Colorimetric measurement was performed under standard conditions at the medial and lateral parts of the scar, the scar at the center of the inframammary fold and at the nipple (at the topmost point). Normal skin was measured at each time point in each patient and subtracted on the values of the different sides of the scars.

Statistical analysis was performed using ANOVA with time and smoking as independent variables. Chi-square testing was used for statistical analysis of wound dehiscence between both groups. SPSS for Mac version 16 UK was used for analysis. Values are presented as means \pm STD.

\section{Results}

Forty-nine patients presented for reduction mammaplasty at the two centers. Three patients were excluded because of diabetes and/or corticosteroid use. Three other patients were excluded because no follow-up took place during scheduled data at the outpatient clinic. The study sample size was 43 patients.

There were 13 smokers and 30 nonsmokers in this group. The mean age of the smokers and nonsmokers was 32.6 years $( \pm$ STD 12.7$)$ and 37.4 years $( \pm$ STD 13.2). There were no differences in mean body mass index (BMI) or resection weight between the two groups (Table 1). Antibiotics were given postoperatively in four patients from the smoking group and in seven patients from the nonsmokers because of signs of inflammation (fever, redness, pain and edema of the breast). Silicone dressings were used in two smoking patients and in eight nonsmokers because hydration was not sufficient and signs of hypertrophy arose.

The incidence of any postoperative dehiscence was higher $(p<0.05)$ in the smoking group compared to the nonsmoking patients $(69.2 \%$ versus $36.6 \%$, Table 1$)$.

The redness of scars in both groups decreased over time, mainly in the center of the inframammary fold and at the nipple. In addition, the scars of the smokers were significantly less red compared to the nonsmokers. This was seen at the

Table 1 Patient characteristics

\begin{tabular}{llll} 
& & Nonsmokers $(n=30)$ & Smokers $(n=13)$ \\
\cline { 2 - 4 } & Age (years) & $37.4($ STD 13.2) & $32.6(\mathrm{STD} 12.7)$ \\
& Body mass index $\left(\mathrm{kg} / \mathrm{m}^{2}\right)$ & $26.1(\mathrm{STD} 3.1)$ & $26.3(\mathrm{STD} 3.1)$ \\
& Resection weight $(\mathrm{g})$ & $436.8(\mathrm{STD} 197.3)$ & $438.5(\mathrm{STD} 180.4)$ \\
& Wound dehiscence $(\%)$ & $36.6 \%{ }^{*}$ & $69.2 \%{ }^{*}$ \\
${ }^{*} p<0.05$ (statistical significance) & Drain production $(\mathrm{ml})$ & $26,3(\mathrm{STD} 19.2)$ & $22.6(\mathrm{STD} 20.9)$ \\
\cline { 3 - 4 } & & &
\end{tabular}



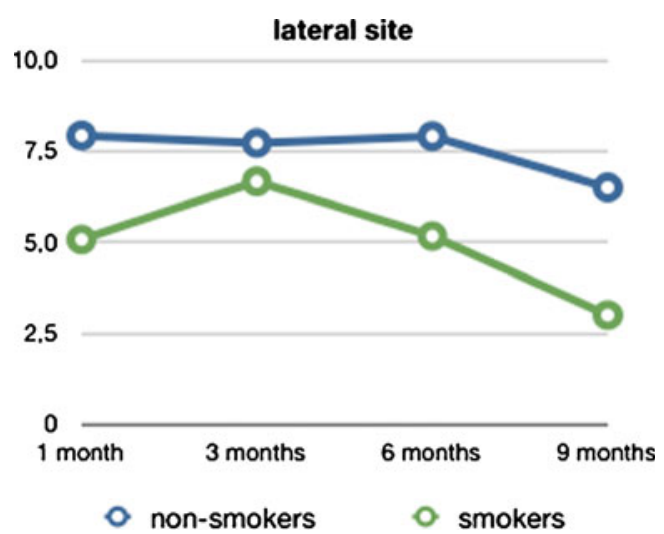

Fig. 1 Redness measured postoperatively with the colorimeter at the lateral part of the scar. Significant difference in redness after 1 month $(p<0.01)$ and after 9 months $(p<0.05)$

lateral site after 1 month $(p<0.01)$ and 9 months $(p<0.05)$, at the medial site after 3 months $(p<0.005)$ and at the nipple after 1 month $(p=0.01)$ and 9 months $(p<0.05)$ (Figs. 1-3). At the center of the mammary fold, no significant differences were found during the period of follow-up (Fig. 4).

\section{Discussion}

This study was conducted to evaluate the effects of smoking on wound healing and scar formation assessed by erythema measurements. The clinical impression that smokers, although having increased risk of developing wound complications, have better cosmetical appearing scars and a diminished risk of hypertrophic scar formation was confirmed by demonstrating decreased vascularity reflected by the diminished redness of the scar. Although the number of patients in the smoking group is small, the diminished redness in this group was statistically clearly demonstrated. It has been shown that the presence of scar

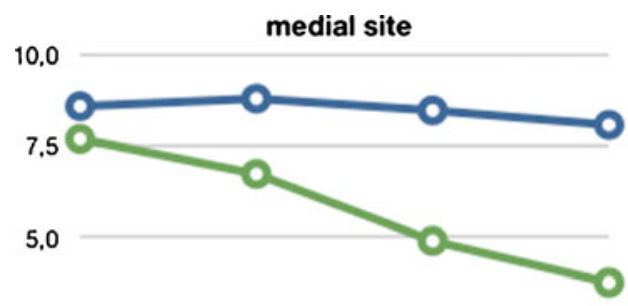

2,5

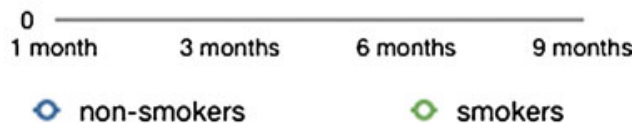

Fig. 2 Redness measured postoperatively with the colorimeter at the medial part of the scar. Significant difference after 3 months $(p<0.005)$. After 6 months, there was a slight difference in redness $(p=0.066)$

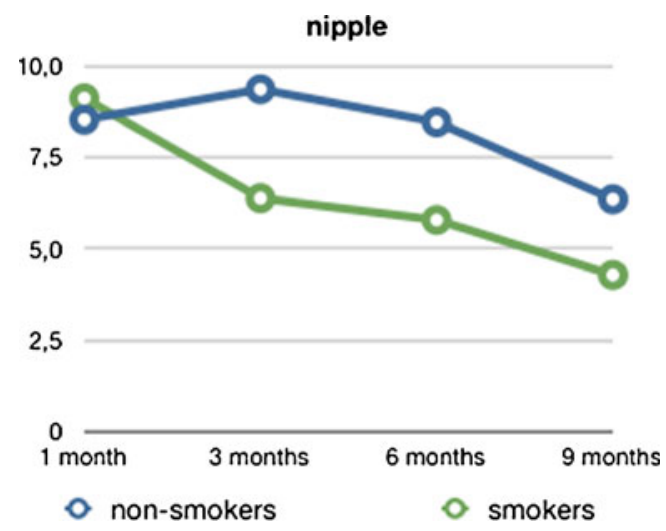

Fig. 3 Redness measured postoperatively with the colorimeter at the topmost point of the nipple. Significant difference after 1 month $(p<0.01)$ and after 9 months $(p<0.05)$

redness is correlated with the occurrence of hypertrophy. Moreover, it has been considered to be associated with the degree of elevation, hardening and retraction of the scar [5]. Smoking, therefore, might influence the risk of hypertrophy. Our hypothesis is that the mechanism by which this happens is the same as the one responsible for the increased wound complication rate in smokers: a deterioration of proliferative and/or immunological response.

Smoking is one of the most important causes of perioperative complications [6]. Postoperative complication rates among smokers varies from 3.1 to $19 \%$ [7-10]. Most of these wound complications consist of the development of a postoperative infection and hematoma formation [6]. In a well-performed recent study in breast reduction surgery, the effect of smoking on wound complications was demonstrated. An incidence of $40 \%$ in postoperative complications was seen among smokers proven by cotinine levels in urine compared to $12 \%$ in nonsmokers [11]. In our study, the overall incidence of postoperative complications was

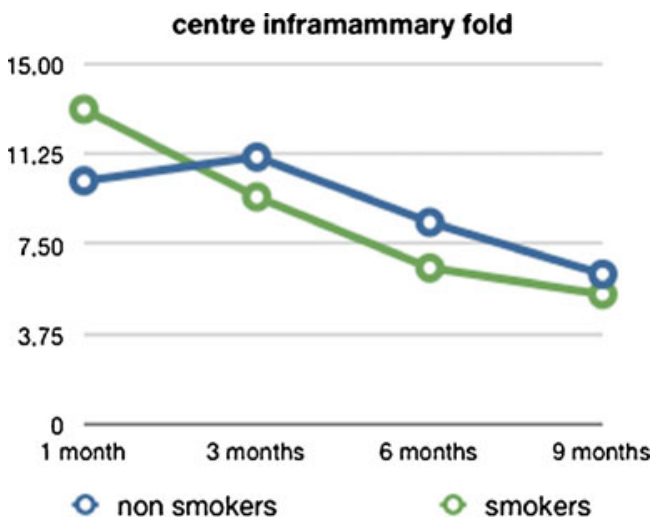

Fig. 4 Redness measured postoperatively with the colorimeter at the center of the inframammary fold. No significant differences were found in the scar of the nipple. The differences in redness are $p=0.116$ after 1 month, $p=0.209$ after 3 months, $p=0.107$ after 6 months and $p=0.575$ after 9 months 
relatively high. This is explained by the definition of wound complications, which was defined quite broadly in our study. Any wound defect until 3 months after surgery, independent on size including, e.g., spitting of suture material was defined as a wound complication. It is obvious that measurement of redness was not performed at the sites were there was or had been a wound dehiscence.

The pathological mechanism of an increased risk for postoperative wound complications due to smoking can be explained in several ways. One possible mechanism could be through the changed availability of nitric oxide as is hypothesized in the association between smoking and vascular damage. Another possibility could be an altered inflammatory response. Sorensen et al studied the effect of smoking on wound healing [12]. The influx of macrophages is shown to be less in smokers. Macrophages produce proinflammatory cytokines that control adhesion and migration of inflammatory cells. Their subsequent proliferation is triggered by multiple growth factors such as PDGF, EGF, TGF- $\beta$, and cytokines IL-1 and TNF. A diminished number of macrophages result in a disturbed cascade of this inflammatory response.

Interestingly, the consequences of smoking on cytokines and growth factors resulting in an increased risk of wound complications may have a beneficial effect on scar formation, resulting in cosmetically superior, less active scars. It has been well established that in a normal situation TGF- $\beta$ causes fibroblast migration and proliferation, increased synthesis of collagen and a decreased degradation of extra cellular matrix (ECM) by metalloproteinases needed for normal wound healing. In adults, the subclasses TGF- $\beta_{1}$ and TGF- $\beta_{2}$ are mainly responsible for this effect. In fetal healing, there is a low expression of TGF- $\beta_{1}$ and TGF- $\beta_{2}$ and increased expression of TGF- $\beta_{3}$ associated with less visible scars $[3,13]$. Therefore, the relative concentrations of the several subclasses of TGF- $\beta$ seem to play an essential role in the appearance of scars. Smoking has been shown to have an inhibiting effect on the influx of macrophages, the initiators of the inflammatory cascade and the subsequent production of TGF- $\beta$. The hypothesis is that the relative contribution of TGF- $\beta_{1}$ is less in wounds of smokers, resulting in increased risk of complicated wound healing but also resulting in cosmetically better looking scars. In this line, it has been shown that serum levels of TGF- $\beta_{1}$ in nicotine-treated rats versus saline-treated rats showed a significantly reduced serum level of TGF- $\beta_{1}[14]$.

In conclusion, we hypothesize that smoking alters the inflammatory response resulting in more wound complications but also less active scarring. Without doubt, smoking has severe adverse effects and creates a strong addiction Therefore, it is absolutely not recommended as treatment. However, our observations give rise to further exploration of the pathofysiological effect that smoking and its specific components may have on wound healing and scar formation. This may eventually result in new ways to treat scars and prevent wound complications.

\section{Conflicts of interest None}

Open Access This article is distributed under the terms of the Creative Commons Attribution Noncommercial License which permits any noncommercial use, distribution, and reproduction in any medium, provided the original author(s) and source are credited.

\section{References}

1. Eming SA, Hammerschmidt M, Krieg T, Roers A (2009) Interrelation of immunity and tissue repair or regeneration. Semin Cell Dev Biol 20(5):517-27.1

2. Falanga V (2005) Wound healing and its impairment in the diabetic foot. Lancet 366(9498):1736-43

3. van der Veer WM, Bloemen MCT, Ulrich MMW et al (2009) Potential cellular and molecular causes of hypertrophic scar formation. Burns 35(1):15-29

4. Bock O, Schmid-Ott G, Malewski P, Mrowietz U (2006) Quality of life of patients with keloid and hypertrophic scarring. Arch Dermatol Res 297(10):433-8

5. Van den Kerckhove E, Stappaerts K, Fieuws S et al (2005) The assessment of erythema and thickness on burn related scars during pressure garment therapy as a preventive measure for hypertrophic scarring. Burns 31(6):696-702

6. Wein RO (2009) Preoperative smoking cessation: impact on perioperative and long-term complications. Arch Otolaryngol Head Neck Surg 135(6):597-601

7. Cardenas-Camarena L (2009) Reduction mammoplasty with superolateral dermoglandular pedicle: details of 15 years of experience. Ann Plast Surg 63(3):255-61

8. Beer GM, Spicher I, Cierpka KA, Meyer VE (2004) Benefits and pitfalls of vertical scar breast reduction. Br J Plast Surg 57(1):12-9

9. Scott GR, Carson CL, Borah GL (2007) Dermabond skin closures for bilateral reduction mammaplasties: a review of 255 consecutive cases. Plast Reconstr Surg 120(6):1460-5

10. Thoma A, Sprage S, Veltri K, Duku E, Furlong W (2007) A prospective study of patients undergoing breast reduction surgery: health-related quality of life and clinical outcomes. Plast Reconstr Surg 120(1):13-26

11. Bartsch RH, Weiss G, Kastenbauer T, Patocka K, Deutinger M, Krapohl BD, Benditte-Klepetko HC (2007) Crucial aspects of smoking in wound healing after breast reduction surgery. J Plast Reconstr Aesthet Surg 60(9):1045-9

12. Sorensen LT, Toft B, Rygaard J, Ladelund S, Teisner B, Gottrup F. Smoking attenuates wound inflammation and proliferation while smoking cessation restores inflammation but not proliferation. Wound Repair Regen. 18(2): p. 186-92.

13. Ferguson MWJ, Duncan J, Bond J et al (2009) Prophylactic administration of avotermin for improvement of skin scarring: three double-blind, placebo-controlled, phase I/II studies. Lancet 373(9671):1264-74

14. Breivik T, Gundersen Y, Giermo P, von Horsten S, Opstad PK (2009) Nicotine acethylcholine receptor activation mediates nicotine-induced enhancement of experimental peridontitis. J Peridontal Res 44(1):110-6 